\title{
Sublinguale Immuntherapie: Hilfreich oder nicht?
}

\section{Die Zahl der Untersuchungen zur sublingualen Immuntherapie nimmt zu. Der Trend weist dabei in Richtung eines positiven Wirksamkeitsnachweises - die Etablierung standardisierter Therapieschemata läßt aber noch auf sich warten.}

Die Immuntherapie mit ihrer klassischen Allergenapplikation in Form von subkutanen Injektionen ist allgemein anerkannt in der Allergietherapie. Noch vergleichsweise wenig Studien liegen jedoch vor zur Effektivität und Sicherheit der Hyposensibilisierung mit sublingualer Verabreichung der Allergene. Neues, wenn auch nicht endgültig Überzeugendes hierzu war auf dem diesjährigen Kongreß der European Academy of Allergology and Clinical Immunology in Brüssel/Belgien zu erfahren.

\section{Negative und ...}

So wartete J. M. Abreu Nogueira, Lissabon/Portugal, mit zunächst enttäuschenden Ergebnissen auf. Er berichtete von der Zwischenauswertung einer doppelblinden, plazebokontrollierten Studie an 22 Kindern im Alter zwischen 8 und 15 Jahren, bei denen sich eine Hausstaubmilben-Allergie überwiegend in einer nasalen Symptomatik manifestierte. Sie erhielten ein standardisiertes Hausstaubmilben-Allergenpräparat (5000 UT/ml) oder Plazebo. Die Auswertung der Parameter Selbsteinschätzung, Einschätzung durch den behandelnden Arzt und Verbrauch an symptomatisch wirkenden Medikamenten nach einem Jahr sublingualer Behandlung zeigte keine Unterschiede zwischen der Verum- und Plazebogruppe.

Doch die portugiesische Arbeitsgruppe will weitermachen: Zum einen könnte der einjährige Beobachtungsintervall zu kurz gewesen sein, um ein positives Resultat zu erzielen, zum anderen ist die verabreichte Dosis möglicherweise zu gering gewesen: Immerhin wurden bei publizierten, anerkann- ten Studien zur sublingualen Immuntherapie, die eine signifikante Verbesserung der Symptomatik ergaben, fünf- bis zwanzigfach höhere Dosen als bei der subkutanen Immuntherapie verwendet.

\section{... positive Ergebnisse}

Mit positiven Resultaten warteten dagegen 2 Studien auf, die als Poster präsentiert wurden. Eine holländische $\mathrm{Ar}$ beitsgruppe (R. A. Luwema et al.) berichtete von der Zwischenauswertung einer Untersuchung an erwachsenen Patienten mit einer HausstaubmilbenAllergie, bei denen doppelblind gegen Plazebo die Wirksamkeit eines standardisierten Hausstaubmilben-Extrakts (700 BU/ml) über eine zweijährige Behandlungsdauer getestet wurde. Nach einer dreiwöchigen Initialphase nahMonate vollkommen verschwunden. men die Patienten zweimal wöchentlich $1 \mathrm{ml}$ der Allergenzubereitung sublingual. Die Auswertung der Daten von 56 Patienten ergab eine Wirksamkeit der sublingualen Immuntherapie, gemessen an dem Parameter der täglichen Symptome, wobei der Effekt im Verlauf der Therapie zunahm.

Noch deutlicher waren die Ergebnisse einer Anwendungsbeobachtung, vorgestellt von V. Di Rienzo et al. aus Italien. 271 Kinder im Alter zwischen 2 und 15 Jahren - überwiegend sensibilisiert gegen Hausstaubmilben und mit allergischer Rhinitis mit und ohne Asthma - erhielten durchschnittlich 28 Monate lang standardisierte Allergene in wässriger Lösung (Pangramin SLIT). Insgesamt ergab sich bei über $80 \%$ der Kinder nach 2 Jahren eine positive Wirkung (sehr gutes oder gutes Therapieergebnis). Die Verträglichkeit war sehr gut - ein wichtiges Kriterium für die Behandlung bei Kindern und hier erstmals an einem größeren Patientenkollektiv dokumentiert.

Fazit: Die sublinguale Immuntherapie scheint wirksam zu sein - wenngleich auch weiterhin Standards in der Dosierung, der Therapiedauer und in der Patientenauswahl noch nicht in Sicht sind.

Vorträge und Poster präsentiert anläßlich des Annual Meeting f the European Academy of Allergology and Clinical Immunology, Brüssel/Belgien, 1999 .

\section{Sublinguale Immuntherapie: Ein Fallbericht}

Daß die sublinguale Immuntherapie im Einzelfall zu guten Ergebnissen kommt, zeigt der Fallbericht einer 21jährigen Patientin aus Kroatien mit einer perennialen Rhinokonjunktivitis aufgrund einer allergologisch nachgewiesenen Hausstaubmilben-Allergie. Die sublinguale Hyposensibilisierung wurde mit einer 20 Wochen dauernden Initialtherapie begonnen, bei der in ansteigender Dosierung (50, 500, 5000, 50000 SQ-E/ml) standardisierter Allergenextrakt ALK-Epipharm gegeben wurden. Die anschließende Erhaltungstherapie lief über 18 Monate. Lediglich zu Beginn der Einnahmephase unter der höchsten Dosierung verspürte die Patientin ein Jucken auf der Zunge und mußte ständig niesen, während sie die Allergene applizierte. Später wurde die Medikation problemlos vertragen.

Bereits nach 6 Behandlungsmonaten wies die Patientin bei Pricktest und IgE-spezifischem Allergen eine deutliche Reduktion der zuvor pathologischen Werte auf - ein Trend, der sich bis zum Ende der Behandlung verstärkte. Und vor allem wichtig: Die klinischen Symptome waren nach 18 